\title{
Treatment of Elizabethkingia meningoseptica Neonatal Meningitis with Combination Systemic and Intraventricular Therapy
}

\author{
Preetha Joshi $^{1} \cdot$ Bhavya Shah ${ }^{1} \cdot$ Vinay Joshi $^{1} \cdot$ Abhaya Kumar $^{2} \cdot$ Tanu Singhal $^{3}$
}

Received: 20 August 2018 / Accepted: 17 January 2019 / Published online: 21 February 2019

(C) Dr. K C Chaudhuri Foundation 2019

\begin{abstract}
Elizabethkingia meningoseptica, a gram negative bacillus ubiquitous in the hospital environment, is known to infrequently cause serious neonatal infections, particularly meningitis which is associated with high mortality and neuromorbidity in survivors. The authors describe a healthy term newborn with no apparent risk factors who developed Elizabethkingia meningoseptica sepsis and meningitis on day 6 of life. Diagnosis could be established only after a week of the illness by which time the baby developed refractory status epilepticus, ventriculitis and hydrocephalus. The isolate was susceptible to only ciprofloxacin, tigecycline and rifampicin and resistant to vancomycin. Apart from systemic combination therapy for $12 \mathrm{wk}$, intraventricular vancomycin was given through an external ventricular drain for $4 \mathrm{wk}$ and later a ventriculo-peritoneal (VP) shunt was inserted. With this regime, authors demonstrated microbiologic and clinical cure. The baby is neurologically normal over a 6 mo follow-up.
\end{abstract}

Keywords Elizabethkingia $\cdot$ Meningitis $\cdot$ Intraventricular $\cdot$ Vancomycin $\cdot$ Neonate

\section{Introduction}

Elizabethkingia meningoseptica, previously known as Chryseobacterium meningosepticum till 2005 and Flavobacterium meningosepticum till 1994 is an ubiquitous Gram negative bacillus colonizing the hospital environment including sinks, water tanks, incubators, beds, saline solutions, syringes, feeding tubes, ventilator tubings, humidifiers among many other sites [1]. It has been associated with serious infections in newborns, patients in the intensive care unit and the immunocompromised. Newborn disease is predominantly meningitis, more common in the premature and often occurs as outbreaks [2]. Accurate diagnosis and susceptibility testing requires advanced microbiologic methods. Treatment

Tanu Singhal

tanusinghal@yahoo.com; tanu.singhal@relianceada.com

1 Department of Neonatal Critical Care, Kokilaben Dhirubhai Ambani Hospital and Medical Research Institute, Four Bungalows, Andheri West, Mumbai 400053, India

2 Department of Neurosurgery, Kokilaben Dhirubhai Ambani Hospital and Medical Research Institute, Four Bungalows, Andheri West, Mumbai 400053, India

3 Department of Pediatrics, Kokilaben Dhirubhai Ambani Hospital and Medical Research Institute, Four Bungalows, Andheri West, Mumbai 400053, India is challenging since the organism is resistant to most antimicrobials used for empirical therapy in neonatal sepsis/ meningitis including beta lactams, carbapenems and aminoglycosides [3]. A recent review described a total of 283 published cases of Elizabethkingia infections in children, of which 35 cases were from India and more than three quarters were in neonates. Neonatal mortality was $37 \%$ and nearly $1 / 3$ rd of survivors had sequelae including hydrocephalus and other deficits [2].

The authors' share their experience of a newborn with severe Elizabethkingia meningitis that was successfully treated with prolonged intraventricular vancomycin therapy despite the isolate being resistant to it in vitro.

\section{Case Report}

A 6-d-old male, exclusively breastfed baby presented to another hospital with fever, vomiting and irritability of $1 \mathrm{~d}$ duration. $\mathrm{He}$ was born at term to a fourth gravida with two previous first trimester abortions by elective lower segment Cesarean section at a small maternity home with a birth weight of $3.5 \mathrm{~kg}$. The baby was roomed in with the mother and exclusively breastfed and discharged at day 4 of life. There were no invasive procedures performed on the baby. At admission, the baby was sick looking but sepsis screen was negative. Treatment with 
intravenous cefotaxime and amikacin was initiated, but the symptoms persisted and by day 13 of life the baby developed seizures. A repeat sepsis screen showed a rise in WBC count and c-reactive protein(CRP). Cerebrospinal fluid (CSF) analysis was suggestive of bacterial meningitis with 2800 cells, $90 \%$ polymorphs, protein of $266 \mathrm{mg} / \mathrm{dl}$ and sugar of $36 \mathrm{mg} / \mathrm{dl}$ against a random blood sugar of $98 \mathrm{mg} / \mathrm{dl}$. Antibiotics were upgraded to meropenem and the baby was referred to authors' hospital. The baby was intubated, ventilated and managed for refractory status epilepticus. The MRI Brain showed signs of meningitis, ventriculitis and hydrocephalus (Fig. 1). The lumbar puncture was repeated; both this CSF and the previous one grew Gram negative rods identified as Elizabethkingia meningoseptica by VITEK 2 and MALDI-TOF MS. The isolate was sensitive only to ciprofloxacin, tigecycline and rifampicin. The vancomycin MIC by E strip was 16 (susceptibility breakpoint 4). Treatment with intravenous ciprofloxacin ( $10 \mathrm{mg} / \mathrm{kg}$ twice daily), tigecycline $(1.5 \mathrm{mg} / \mathrm{kg}$ twice daily), vancomycin $(15 \mathrm{mg} / \mathrm{kg}$ thrice daily) and oral rifampin $(10 \mathrm{mg} / \mathrm{kg} / \mathrm{d})$ was initiated. An external ventricular drain (EVD) was placed and vancomycin $10 \mathrm{mg}$ was given daily through EVD followed by clamping of the drain for $6 \mathrm{~h}$. Trough CSF vancomycin level was 76 microgram $/ \mathrm{ml}$. The baby improved, the CSF became culture negative $7 \mathrm{~d}$ after initiating therapy and the EVD removed after $2 \mathrm{wk}$ of therapy. Soon after, the head circumference increased and baby became drowsy. Repeat MRI Brain showed increased hydrocephalus with a new infarct in the right thalamus. The EVD was re-introduced and intraventricular vancomycin was given for 2 more weeks (though the CSF cultures were sterile from the fresh EVD). The CSF cell count and protein improved over time but did not normalize. A VP shunt was subsequently inserted. Systemic antibiotics were continued for a total period of $6 \mathrm{wk}$ and then oral ciprofloxacin and rifampicin continued for another $6 \mathrm{wk}$. At nine mo follow- up the baby is physically and neurodevelopmentally normal.

\section{Discussion}

This baby with early onset sepsis with Elizabethkingia meningoseptica probably acquired the infection from exposure to contaminated water at the maternity home. Since this pathogen is an uncommon cause of neonatal sepsis, the initial
Fig. 1 MRI brain showing meningitis, ventriculitis and hydrocephalus

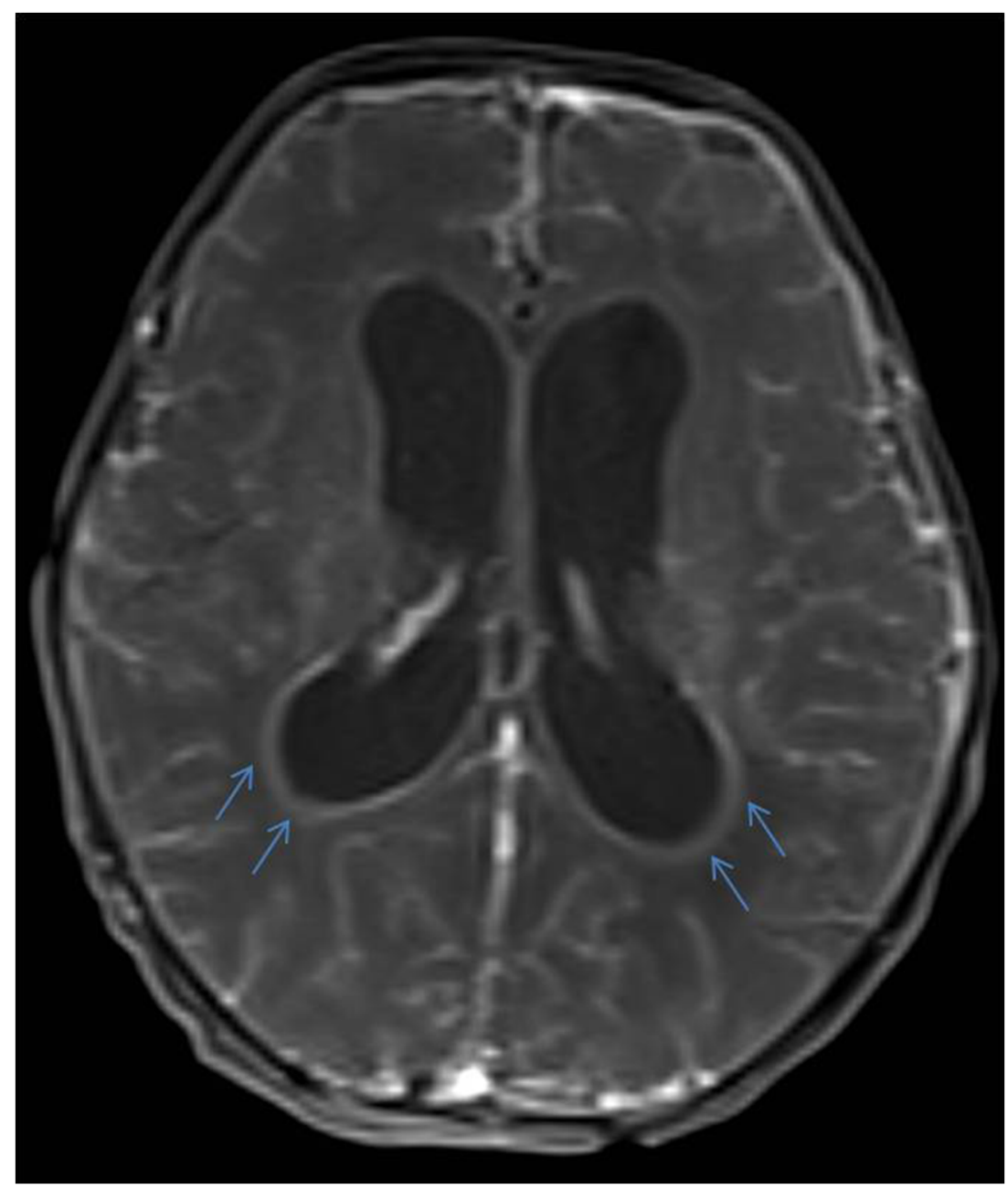


empiric antimicrobial therapy employed in this case and that in usual practice were ineffective.

Elizabethkingia meningoseptica demonstrates in vitro resistance to many antibiotics [3]. There are no established CLSI breakpoints. Disc diffusion testing, VITEK and E strip are known to be inaccurate for susceptibility testing; broth microdilution is the preferred method [4]. Drugs known to be active and used successfully for therapy include cotrimoxazole, tigecycline, minocycline, quinolones, piperacillin, tazobactam, cefepime and drugs active against gram positive bacteria such as vancomycin and rifampicin [4-8]. There have however been reports of resistance, unreliable susceptibility and failure of vancomycin in treatment of Elizabethkingia infections [9].

Treatment with multiple drugs is not the usual norm in Gram negative meningitis. However multiple drugs were used in the index case for the following reasons 1) the newborn was extremely sick with impending risk of death or serious neurologic morbidity 2) results of susceptibility testing by VITEK are often unreliable 3) Combination antimicrobial therapy is recommended for Elizabethkingia 4) the CNS penetration of the some of the drugs used (tigecyline) is limited. The isolate in index patient was resistant to vancomycin, a drug which has been used with fair degree of success in previous reports. However, there was a possibility that the susceptibility results for vancomycin by VITEK/ E strip were inaccurate (The authors did not have access to broth microdilution). Hence vancomycin was used despite in vitro resistance. Further, it is possible to achieve high levels of drug with local therapy. Standard guidelines also recommend use of local intraventricular/ intrathecal therapy in resistant meningitis/ ventriculitis [10]. Successful treatment of Elizabethkingia meningitis with intraventricular vancomycin has been previously described [8] Hence intraventricular vancomycin was used in addition to systemic vancomycin. The duration of therapy was also longer than what is usually recommended for meningitis/ ventriculitis in view of the severity of disease, delayed response, persistent CSF and radiologic abnormalities.

This case highlights the need for appropriate infection control practices to prevent infections with this pathogen, correct identification and susceptibility testing and considering unconventional treatment approaches especially when therapeutic options are limited.

Acknowledgements The authors acknowledge help of Sweta Shah, Department of Microbiology for helping in identification and susceptibility testing and Pradnya Gadgil, Pediatric Neurologist.
Authors' Contributions PJ: Intensive care management and drafting of manuscript; BS: Clinical management, literature search and drafting of manuscript; VJ: Intensive care management and drafting of manuscript; AK: Neurosurgical management and drafting of manuscript; TS: Infectious disease opinion and reviewing the manuscript. TS will act as guarantor for this paper.

\section{Compliance with Ethical Standards}

Conflict of Interest None.

Source of Funding None.

Publisher's Note Springer Nature remains neutral with regard to jurisdictional claims in published maps and institutional affiliations.

\section{References}

1. Jean SS, Lee WS, Chen FL, Ou TY, Hsueh PR. Elizabethkingia meningoseptica: an important emerging pathogen causing healthcare-associated infections. J Hosp Infect. 2014;86:244-9.

2. Dziuban EJ, Franks JL, So M, Peacock G, Blaney DD. Elizabethkingia in children: a comprehensive review of symptomatic cases reported from 1944 to 2017. Clin Infect Dis. 2018;67: $144-9$.

3. Kirby JT, Sader HS, Walsh TR, et al. Antimicrobial susceptibility and epidemiology of a worldwide collection of Chryseobacterium spp.: report from the SENTRY antimicrobial surveillance program (1997-2001). J Clin Microbiol. 2004;42:445-8.

4. Fraser SL, Jorgensen JH. Reappraisal of the antimicrobial susceptibilities of Chryseobacterium and Flavobacterium species and methods for reliable susceptibility testing. Antimicrob Agents Chemother. 1997;41:2738-41.

5. Hsu MS, Liao CH, Huang YT. Clinical features, antimicrobial susceptibilities, and outcomes of Elizabethkingia meningoseptica (Chryseobacterium meningosepticum) bacteremia at a medical center in Taiwan, 1999-2006. Eur J Clin Microbiol Infect Dis. 2011;30: 1271-8.

6. Aykac K, Ozsurekci Y, Tuncer O, et al. Six cases during 2012-2015 and literature review of Chryseobacterium indologenes infections in pediatric patients. Can J Microbiol. 2016;62:812-9.

7. Ghafur A, Vidyalakshmi PR, Priyadarshini K, Easow JM, Raj R, Raja T. Elizabethkingia meningoseptica bacteremia in immunocompromised hosts: the first case series from India. South Asian J Cancer. 2013;2:211-5.

8. Soman R, Agrawal U, Suthar M, Desai K, Shetty A. Successful management of Elizabethkingia meningoseptica meningitis with intraventricular vancomycin. J Assoc Physicians India. 2017;65: 98-9.

9. Jean SS, Hsieh TC, Ning YZ, Hsueh PR. Role of vancomycin in the treatment of bacteraemia and meningitis caused by Elizabethkingia meningoseptica. Int J Antimicrob Agents. 2017;50:507-11.

10. Tunkel AR, Hartman BJ, Kaplan SL, et al. Practice guidelines for the management of bacterial meningitis. Clin Infect Dis. 2004;39: 1267-84. 